\title{
Application of Computational Intelligence Paradigm in the Probabilistic Routing for intermittently connected network.
}

\author{
Jahnabi Borah \\ Department of Computer Science Engineering \& IT, Assam Don Bosco University Azara, Airport Road, \\ Assam.
}

\begin{abstract}
In this project an approach is being made to use Fuzzy Logic(a computational intelligence paradigm) in the Probabilistic routing protocol for Intermittently connected network: Delay Tolerant Network. Communication between two mobile nodes in an infrastructure less network is possible if they are within the wireless communication range. Delay Tolerant Network (or DTN) is not only an infrastructure less network but it also suffers from intermittent connection i.e. there is not always a connected path from the source to the destination. As a result traditional routing protocols cannot serve the purpose of this type of network. A passive routing protocol for DTN's named PROPHET routing works on the principle of history of encounters and transitivity [3]. It is called Probabilistic routing protocol since it uses a parameter 'Delivery of Predictability'. Using Fuzzy Logic some rules will be defined and another parameter 'Energy value' will be considered to calculate the probability of delivery. With the aim of consuming fewer resources by the network and minimizing the delay in message delivery this new Fuzzy based probabilistic routing protocol has been introduced.
\end{abstract}

Keywords: Delay tolerant network, Fuzzy logic, Delivery Predictability, Prophet routing protocol, Epidemic routing protocol.

\section{INTRODUCTION}

Delay tolerant network (DTN) is very similar to Mobile Ad Hoc network(MANET) only with a difference that there is no directly connected path between the source and the destination or even via intermediate routers. Like MANETS, DTN is also infrastructure less and so all nodes participating in such network has to act as a host and also as a router. Thus the routing protocols used in MANETS cannot be use in this type of partially connected network. Various routing protocols have been proposed for DTNs[5], such as

1. Deterministic Routing Protocols

Oracle based, Link state based, Space time based.[5]

\section{Stochastic Routing Protocols}

\subsection{Passive Routing}

Epidemic routing [2], PROPHET [3], Spray Wait [4] and many more.

\subsection{Active Routing}

Meet and Visit, Message Ferrying.[5]

These routing protocols follow the "store-carry-forward" routing mechanism and so as to increase the message delivery ratio. The delay tolerant networking research group (DTNRG) defined the Delay tolerant network as "How to address the architectural and protocol design principles arising from the need to provide interoperable communications with and among extreme and performance challenged environments where continuous end to end connectivity cannot be assumed".

Routing in DTN is totally different from routing in MANETS because there is frequent disconnections. So while designing a routing protocol for DTN, we have to take into consideration the following characteristics of DTN such as [5]:

- Intermittent connection

- Energy Constraint

- Delay in message delivery

- Long queuing of message

- Limitation of resource

- Limited Longevity

- Security

With the aim of consuming less resources like buffer, energy of the node by the network and minimizing the delay of message delivery, a routing approach has been proposed to improve the PROPHET 
routing protocol based on two routing metrics: History of contact between the mobile nodes[3] and the battery capacity of each node[6].

\section{Epidemic Protocol}

\section{RELATED WORKS}

It was the method introduced by Demers et al. in to synchronize databases which use replication mechanism [5]. This algorithm was modified by Vahdat et al. (2000) and proposed as a flooding based forwarding algorithm for DTNs[5].

In [2], Vahdat et al. developed techniques to deliver messages in the case where there is absence of a connected path from source to destination. Whenever a node receives a message, it forwards a copy of it to all nodes it meets on its way. Thus the message is spread throughout the network by mobile nodes and eventually it will reach the destination. Each message is saved in the node's buffer and has a unique identifier called summary vector [2]. Whenever two nodes meet each other, they exchange and compare their summary vectors to identify which messages they do not have and subsequently exchange them. The overall goal of Epidemic Routing is to maximize message delivery rate and minimize message delivery latency, while also minimizing the aggregate system resources consumed in message delivery [2]. They implemented their protocol in the Monarch [7] simulator.

The disadvantage of this protocol is that it wastes resources such as buffer, bandwidth and nodes power due to forwarding of multiple copies of the same message. The epidemic protocol can be use in those scenarios when there is no better algorithms to deliver messages.

\section{PROPHET protocol.}

PROPHET (Probabilistic Routing Protocol using History of Encounters and Transitivity) [3] is a routing protocol developed by Lindgren et al in 2003. PROPHET works somewhat similar to epidemic routing protocol but it does not forward the copy of the message to all the nodes that it encounters rather it forwards the copy to only selected nodes. Thus it tries to minimize the use of resource and still attempts to preserve the bestcase routing capabilities of epidemic routing.

In [3] they introduced a metric called Delivery Predictability, $\mathrm{P}(\mathrm{A}, \mathrm{B}) \in[0$, I] i.e. $0<=\mathrm{P}(\mathrm{A}, \mathrm{B})<=1$, is computed at every node A for each known destination B. In PROPHET a node which has a higher delivery predictability value for a particular destination is assumed to be a better router for delivering a message to that destination (i.e., if $\mathrm{P}(\mathrm{A}, \mathrm{B})>\mathrm{P}(\mathrm{C}, \mathrm{B})$, message for destination $\mathrm{B}$ are preferable to forward to $\mathrm{A}$ rather than $\mathrm{C}$.

As routes in a DTN are not symmetric, the calculation of the delivery predictability $\mathrm{P}(\mathrm{A}, \mathrm{B})$ may be different from $\mathrm{P}(\mathrm{B}, \mathrm{A})$. When two nodes meet they update the delivery predictability for each other.

If node $\mathrm{B}$ has not met node $\mathrm{A}$ for a long time or has never met node $\mathrm{B}$, such that $\mathrm{P}(\mathrm{A}, \mathrm{B})<\mathrm{P} \_$threshold, then P_A,B) should be set to P init. P init should be set to 0.5. [3]

The calculation of the probability of delivery is done in three steps[3][1]:

- When a node A meets another node B: A updates the probability of delivery as shown in the following equation:

$\mathbf{P}(A, B)=\mathbf{P}(A, B)$ old $+(1-\partial-P(A, B)$ old $) * P$ init

The value of $\partial$ should normally be very small (e.g., 0.01).

- For a node $\mathrm{C}$ known by a node B: A updates the probability of delivery in accordance with the following equation:

$\mathbf{P}(\mathbf{A}, \mathbf{C})=\max (\mathbf{P}(\mathbf{A}, \mathbf{C})$ old, $\mathbf{P}(\mathbf{A}, \mathbf{B}) * \mathbf{P}(\mathbf{B}, \mathbf{C}) * \beta)$ where $0<=\beta<=1$ is a constant that determines the impact of transitivity on the delivery predictability.

- For nodes infrequently met by A: A updates the probability of delivery in accordance with the following equation:

$\mathbf{P}(\mathbf{A}, \mathbf{B})=\mathbf{P}(\mathbf{A}, \mathbf{B}) * \gamma^{\wedge} \mathbf{k}$ where $\gamma$ is an aging constant and and $\mathrm{k}$ is the number of time units that have elapsed since the last time the metric was aged.

\section{PROPOSED FUZZY BASED PROBABILISTIC ROUTING}

PROPHET routing protocol will be extended using Fuzzy rules and the two routing metric used in our approach is probability of delivery (based on history of encounters)and energy value (battery capacity)which together gives us the Delivery of predictability. 


\section{Fuzzy logic}

Lotfi Zadeh, a professor at the University of California at Berkeley, introduced fuzzy logic. In 1965 he presented the original paper formally defining fuzzy-set theory, from which fuzzy logic emerged. Traditionally, logic has two extreme values: either true (1) or false (0). But, in the fuzzy world, a premise ranges in degree of truth from 0 to 100 percent, which allows it to have a partially true value and a partially false value.[12]

A fuzzy control system involves three steps:

Fuzzification of inputs, Evaluation of rules and Defuzzification of output.[12]

\section{Proposed model}

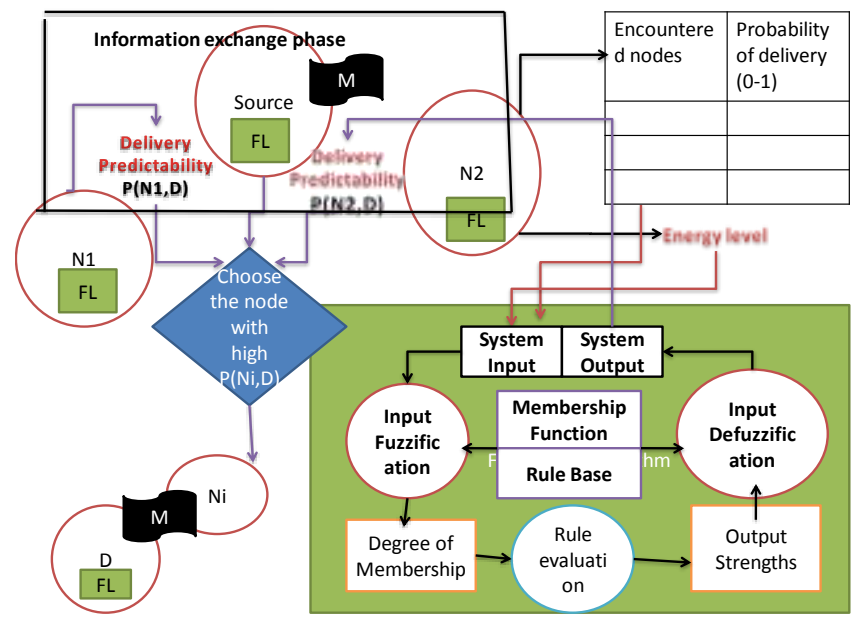

Fig 1: Proposed Fuzzy based probabilistic routing

\section{Fuzzification of Inputs}

In the fuzzification process a crisp input(probability of delivery or energy value) is taken and we determine the degree of membership to which these inputs belong to each of the appropriate fuzzy sets[13] .The crisp input is a numerical input.

The two routing metric used in this approach will be probability of delivery(dp) and the energy value(ev).

Probability of delivery (dp): It uses the history of contact between the mobile nodes. This probability of delivery is calculated using the equations described above. Each node maintains in its cache the information of all the nodes it has contacted recently i.e the frequency of encounters with each node and thus a probability of delivery value will be associated.

Suppose Source node $\mathrm{S}$ has a message $\mathrm{m}$ for Destination D. So whenever $\mathrm{S}$ meets any node in its way it checks for the dp value in that encountered nodes. If encountered node has high probability of delivery but not enough energy or low probability of delivery and high energy then it will not be a good router.

Say S meets B. So the relation between the node B with the destination node D can be of three types[6]:

- Friend: B $\leftrightarrow$ D Node B have sent/received a lot of messages to/ from node D. The probability of delivery between them are reasonably high .

- Aquaintance: $\mathrm{B} \rightarrow \mathrm{D}$ or $\mathrm{D} \rightarrow \mathrm{B}$ Node $\mathrm{B}$ have sent/received few messages from node $\mathrm{D}$. The probability of delivery between them are neither too low nor too high.

- Stranger: neither $D \rightarrow B$ nor $B \rightarrow D$ Node $B$ have never sent/received message to/from node $D$. The probability of delivery between each other will be low.

On the basis of the relation between the B and D node : stranger, acquaintance and friend we define three fuzzy sets :high, medium and low, respectively.[6]

There are some standard dp values:

- P init 0.5 medium

- P_threshold 0.1 low

- P_encounter max: 0.7 high

Energy Value or Battery capacity (ev): We defined that every node is in high level which means it has full capacity $(100 \%)$. The node will not be a good router to forward the packets if the energy of it falls below $50 \%$.[6] For energy capacity between $50 \%$ to $100 \%$ of total capacity, we define high fuzzy set, for $20 \%$ to $80 \%$ we define medium fuzzy set and for $0 \%$ to $50 \%$ we define low fuzzy set. 


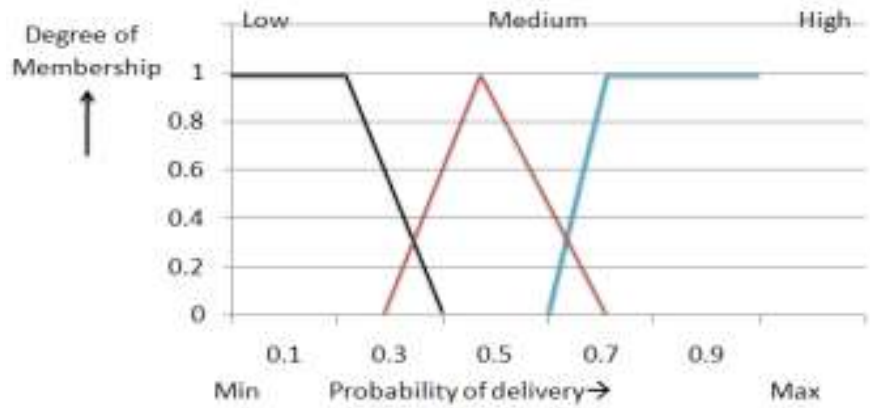

Fig 2: Membership function for probability of delivery

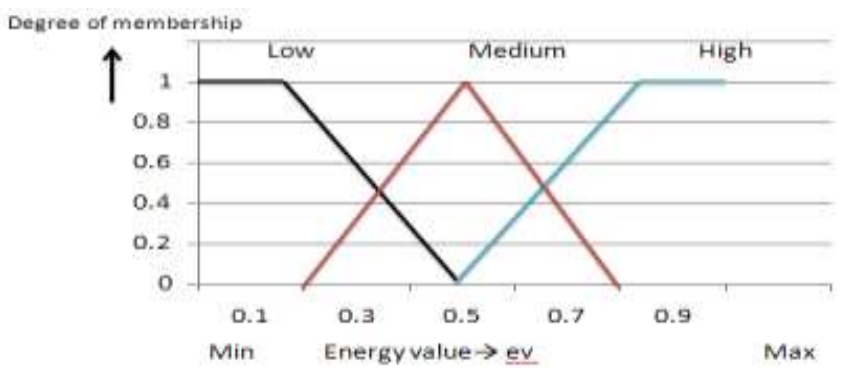

Fig 3: Membership function for energy value

The antecedents of rules correspond directly to degrees of membership calculated during the fuzzification process. The consequent part of the rule may also contain linguistic values. This type of rule is easier to obtain from an expert. The fuzzy classifier in such case works as a Mamdani-type fuzzy system (Mamdani, 1977).

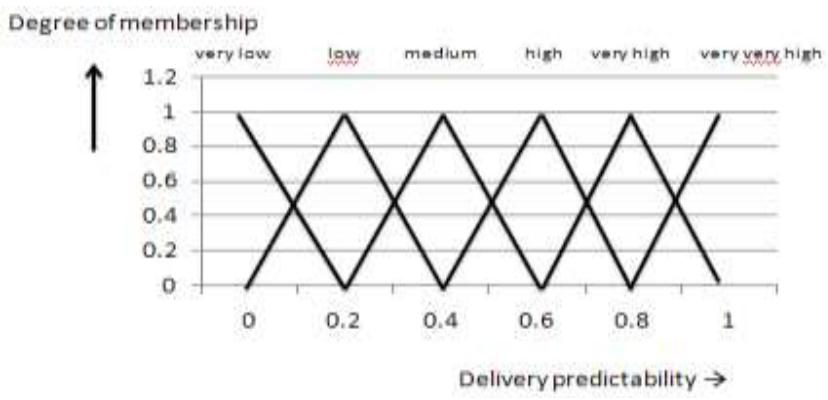

Fig 3: Membership function for Delivery predictability

\section{Evaluation of rules}

To control the system's behaviour, we have to develop a set of rules that have the form of If-Then statements.

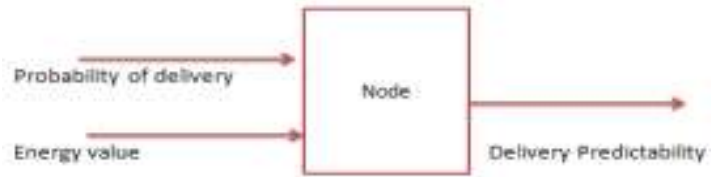

Fig3: Evaluation of fuzzy rules in each node

The two metrics dp and $e v$ are numerical but the rules use linguistic values.

\section{Rule Base}

Cases where the nodes are allowed to transmit message [1]

Rule 1: if dp is high and energy value is high then $\mathrm{P}(\mathrm{N}, \mathrm{D})$ is very very high.

Rule2: if dp is medium and energy value is high then $\mathrm{P}(\mathrm{N}, \mathrm{D})$ is very high.

Rule3: if dp is high and energy value is medium then $\mathrm{P}(\mathrm{N}, \mathrm{D})$ is high.

Rule4: if dp is medium and energy value is medium then $\mathrm{P}(\mathrm{N}, \mathrm{D})$ is medium.

Cases where the nodes are not allowed to transmit message[1]

Rule5: if dp is low and energy value is medium then $\mathrm{P}(\mathrm{N}, \mathrm{D})$ is low. 
Rule6: if dp is medium and energy value is low then $\mathrm{P}(\mathrm{N}, \mathrm{D})$ is very low.

\section{Defuzzification of Outputs}

Fuzzification helps us to evaluate the rules, but the final output of a fuzzy system should be a crisp number instead of a linguistic value. The input for the defuzzification process is the aggregate output fuzzy set and the output is a single number.[8]

One common defuzzification technique, the "center-of-gravity method," consists of several steps. Mamdani-style inference finds the centroid of a two-dimensional shape by integrating across a continuously varying function. In general, this process is not computationally efficient.

In our process the fuzzy system consist of, singleton input fuzzifier, product inference engine and center average defuzzifier [6] and is of the form:

$$
\mathrm{f}(\mathrm{x})=\frac{\sum_{i=1}^{6} y_{0}^{(i)}\left(\prod_{k=1}^{2} \mu_{A_{i k}}\left(x_{k}\right)\right)}{\sum_{i=1}^{6}\left(\prod_{k=1}^{2} \mu_{A_{i k}}\left(x_{k}\right)\right)}
$$

Where $y_{0}^{(i)}$ be the centre of the it fuzzy set, $\left.\prod_{k=1}^{2} \mu_{A_{i k}}\left(x_{k}\right)\right)$ is the height of the ith fuzzy set, $x_{k}$ represents crisp input(probability of delivery or energy value).

\section{CONCLUSION AND FUTURE WORK}

In many areas DTN has found its application. But due to the intermittent connection in these type of network, routing is very challenging. Since traditional ad hoc protocols cannot be implemented in this type of network, so researchers have been trying to introduce DTN specific routing protocol keeping in mind the constraints of these networks. So far a Fuzzy Logic Based Probabilistic routing has been proposed for Delay Tolerant Network. A partially connected network will be designed in Nam of NS2. The PROPHET protocol will be compared with the Fuzzy based probabilistic protocol and a comparison will be done In my next paper simulation study will be performed for packet delivery ratio, throughput, energy consumed and end to end delay evaluations.

Packet delivery ratio: The ratio of packets sent to the successfully received packets.[6][9].

End to End Delay (sec): Average end to end delay is the time taken in the successful delivery of packets from the source to their respective destinations. [6][9].

Throughput (bits/sec): The rate of successfully transmitted data per second in the network. Throughput is the measure of successfully delivered payload sizes of data packets within the period, which starts when a source starts to communicate with a remote destination port. Average throughput can be calculated by dividing total number of bytes received by the total end to end delay [6][9].

Energy spent $(\%)$ : The battery capacity or the energy value that is spent by the nodes in during the simulation time.

On the basis of that result we can conclude whether our proposed approach will perform better than the traditional protocol or not.

\section{REFERENCES}

[ 1 ] EL MASTAPHA SAMMOU “Efficient Probabilistic Routing in Delay Tolerant Networks"2012 IEEE.

[2] A. Vahdat and B. Becker, "Epidemic Routing for Partially-Connecte Ad- hoc Networks," Technical Report CS-2000-06, Duke University,July 2000.

[ 3 ] A. Lindgren, A. Doria, and O. Scheln, "Probabilistic Routing in Intermittently Connected Networks," in Proc. Workshop on Service Assurance with Partial and Intermittent Resources, August 2004.

[ 4 ] T. Spyropoulos, K. Psounis, and C. S. Raghavendra, "Spray and wait: Efficient routing in intermittently connected mobile networks," in Proceedings of ACMSIGCOMM workshop on Delay Tolerant Networking (WDTN), 2005.

[ 5 ] Morteza Karimzadeh "Efficient routing protocol in DTNs",2011

[6] Arash Dana, Golnoosh Ghalavand, Azadeh Ghalavand and Fardad Farokhi "A Reliable routing algorithm for Mobile Adhoc Networks based on fuzzy logic". IJCSI International Journal of Computer Science Issues, Vol. 8, Issue 3, No. 1, May 2011.

[7] CMU Monarch Project. The CMU Monarch Project's wireless and mobility extensions to ns ftp.monarch.cs.cmu.edu/pub/monarch/wireless-sim/ns-cmu.ps, August 1999.

[ 8 ] Olaf Wolkenhauer"Fuzzy Inference Engines Composition and Individual-Rule Based Composition, Non-Linear Mappings".

[9] V.Rishiwal,A.Kush and S.Verma, "Backbone nodes based Stable routing for mobile ad hoc network", UBICC Journal, Vol2,No.3,2007,pp34-39.

[10] Etienne C. R. de Oliveira and C' elio V. N. de Albuquerque "NECTAR: A DTN Routing Protocol Based on Neighborhood Contact History". SAC'09 March 2009.

[11] Peng Wang, Fuke Shen."Method to Improve the Performance of PROPHET Routing Protocol"Second International Workshop on Education Technology and Computer Science, 2010

[12] Greg Viot "Fuzzy Logic in C Creating a fuzzy-based inference engine" Dr. Dobb's Journal , February 1993

[13] Dr Bogdan L. Vrusias "Fuzzy logic 3" b.vrusias@ surrey.ac.uk October 2006 